University of Wollongong

Research Online

Faculty of Engineering - Papers (Archive)

Faculty of Engineering and Information

Sciences

2008

\title{
A dual scintillator - dual silicon photodiode detector module for intraoperative gammalbeta probe and portable anti-compton spectrometer
}

Anatoly B. Rosenfeld

University of Wollongong, anatoly@uow.edu.au

Michael LF Lerch

University of Wollongong, mlerch@uow.edu.au

M. Chan

University of Wollongong

Dean Inwood

University of Wollongong, di03@uow.edu.au

George J. Takacs

University of Wollongong, gjt@uow.edu.au

See next page for additional authors

Follow this and additional works at: https://ro.uow.edu.au/engpapers

Part of the Engineering Commons

https://ro.uow.edu.au/engpapers/622

\section{Recommended Citation}

Rosenfeld, Anatoly B.; Lerch, Michael LF; Chan, M.; Inwood, Dean; Takacs, George J.; Lee, Bong Soo; Gektin, A F.; and Perevertaylo, V L: A dual scintillator - dual silicon photodiode detector module for intraoperative gamma \beta probe and portable anti-compton spectrometer 2008, 458-461.

https://ro.uow.edu.au/engpapers/622 


\section{Authors}

Anatoly B. Rosenfeld, Michael LF Lerch, M. Chan, Dean Inwood, George J. Takacs, Bong Soo Lee, A F. Gektin, and V L Perevertaylo 


\title{
A Dual Scintillator - Dual Silicon Photodiode Detector Module for Intraoperative Gamma $\backslash$ Beta Probe and Portable Anti-Compton Spectrometer
}

\author{
A.B. ROSENFELD ${ }^{1 *}$, M.L.F. LERCH ${ }^{1}$, M. CHAN ${ }^{1}$, D. INWOOD ${ }^{1}$, G.J. TAKACS ${ }^{1}$, D. INWOOD ${ }^{1}$, B. LEE $^{2 * *}$, \\ A.F. GEKTIN ${ }^{3}$, and V.L. PEREVERTAYLO ${ }^{4}$ \\ ${ }^{I}$ Centre for Medical Radiation Physics, University of Wollongong, NSW 2522 Australia \\ ${ }^{2}$ Department of Biomedical Engineering, Research Institute of Biomedical Engineering, \\ College of Biomedical and Health Science, Konkuk University, Korea \\ ${ }^{3}$ AMCRYS-H, Kharkov, Ukraine \\ ${ }^{4}$ SPO BIT, Ukraine
}

\begin{abstract}
A new approach has been investigated for the miniaturization and simplification of intraoperative gamma and beta probes that have recently found application in radioguided surgery and sentinel lymph node biopsy. The probe design that is based on dual annular scintillators coupled to specially designed silicon concentric dual photodiode (CDPD). This approach allows us to avoid fiber optics coupling and PMTs. Two channels readout front-end electronics including shaper amplifier attached directly to the back of the detector module has been proposed. Two geometries of Si dual photodiodes coupled to $\mathrm{CsI}(\mathrm{Tl})$ annular scintillators with light masking between them were fabricated and investigated. CDPDs have size 10 and $6 \mathrm{~mm}$ as well as 6 and $3 \mathrm{~mm}$ outer and inner diameters respectively. The spectroscopy properties of CDPD separately and coupled to CsI(Tl) dual scintillator were investigated on I-125, Co57 and Na-22 photon sources. Both detectors have demonstrated acceptable energy resolution ( $10 \%$ for $511-\mathrm{keV})$ for the proposed application with FDG isotopes. In the smaller detector the amplitude of the $511 \mathrm{keV}$ photopeak from outer detector was less than in inner and is related to the light collection in the current geometry. When used in coincidence mode operation we observe a significant reduction in the measured Compton continuum level relative to the photopeak which is not significantly attenuated. The spatial resolution of detector module measured in the horizontal plane was 2-mm FWHM using a 0.5-mm collimated Tc-99m source.
\end{abstract}

KEYWORDS: photodetector, anti-Compton, lymphoscintigraphy

\section{Introduction}

Intraoperative gamma and beta probes have found application in radioguided surgery and sentinel lymph node biopsy. The development of a portable beta probe suitable for endoscopic procedures is also of great interest. Coupling of the miniature radiation probe with an endoscopic device may eliminate the need of multiple biopsy samples thus providing minimally invasive determination and treatment of cancer.

The most widely used isotopes for cancer targeting are Tc-99m gamma emitter with energy $140 \mathrm{keV}$ and F-18 (FDG) positron emitting isotope with annihilation $511-\mathrm{keV}$ photons. In both cases the finding of the cancer requires the ability of the detection device to distinguish the increased uptake on a radiation background of normal tissue uptake. The situation is even more complicated in the case of FDG tracers because high energy $511-\mathrm{keV}$ photons are producing an essential contribution to the beta channel which makes it difficult to distinguish the spot (often quite small in size) with the highest uptake. The development of unique probe, which will be suitable both for pure gamma emitters and FDG isotopes with excellent spatial resolution, is a challenge.

*Corresponding Author, Tel No: +61-2-42214574, Fax No: +61-242215944, E-Mail; anatoly@uow.edu.au

${ }^{* *} \mathrm{~B}$. Lee is currently a visiting fellow at CMRP on sabbatical
The traditional approach that utilizes passive collimation and scintillator coupled to PMT is not suitable in a $511-\mathrm{keV}$ photon field. Essential increasing of the thickness of the passive protection leads to increasing the size of the probe, making difficult for intraoperative use. Using concentric scintillators separated by layer of lead or steel and coupled to the two separate PMTs via fiber optics demonstrated improvements in the spatial resolution. The improvement was due to the suppression of the 511-keV gamma background in the beta channel (core detector) in case of an FDG isotope or reducing the contribution of scattered gamma radiation to the core detector in case of Tc- $99 \mathrm{~m} .{ }^{1,2)} \mathrm{A}$ detector module for beta activity detection with gamma suppression in case of FDG isotope, which is based on two back-to-back $0.5-\mathrm{mm}$ thick Si SBD detectors with 3-mm diameter of active region, also has been proposed. ${ }^{3)}$ For full depletion of such detectors $150 \mathrm{~V}$ bias is required.

The current paper describes the proposed at CMRP, manufactured and tested prototype of a patented detector module, which will be suitable for gamma/beta activity detection with high spatial resolution on a background of high energy gamma radiation. This module is based on concentric, optically isolated $\mathrm{CsI}(\mathrm{Tl})$ scintillators coupled to a dual single crystal PD, optimized and fabricated for this application.

In this paper we report on the technical characteristics of the dual, circular, concentric photodiodes of different 
geometry that incorporates dual scintillator technology and the spectroscopic characteristics of dual scintillator-dual photodiode module for Co-57 (122 keV), Tc-99m (140 keV), $\mathrm{Na}-22(511 \mathrm{keV})$ and Cs-137 (662 keV). Some comparisons are also made with a currently available commercial probe that is based on a solid state $\mathrm{CdZnTe}$ detector configuration.

\section{Detectors, Materials and Methods}

Fig. 1a and 1b show the principle of the detector module with dual concentric PDs. The advantage of this approach is in identical readout electronics due to the same nature of detectors, possibility of miniaturization with readout miniature front-end electronics attached directly at the back of the detector, low voltage operation $(25 \mathrm{~V})$, utilizing of coincidence technique for improvement of spread function of probe in case of beta/gamma or pure gamma isotope activity mapping.

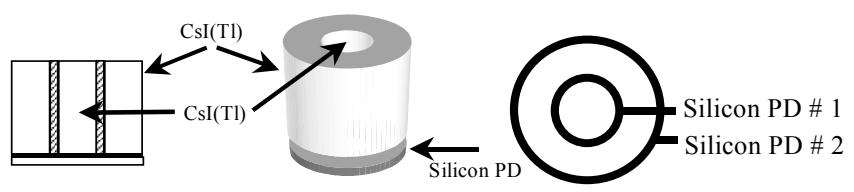

Fig. 1 schematic diagram of (a) the concentric PD coupled to dual scintillator and (b) the concentric PD design

To evaluate the detector module properties, the module was mounted on a computer controlled rotating/translating gantry (shown in Fig. 2a) designed and built at CMRP labs. A lead block as shown in Fig. 2b was used to collimate a variety of radiation sources. In the case of Tc-99m, which was used to determine the spatial resolution of the probe, the radioactive source was sealed in a fine plastic tube, which then positioned inside the block. The probe was positioned $1.9 \mathrm{~mm}$ above the point source defined by the block. The probe was moved in $\mathrm{x}$-direction and acquisition time for the inner detector was $300 \mathrm{~s}$ with Tc-99m (140 keV). Response using the inner detector was measured, with the acquisition time being $30 \mathrm{~s}$. The spatial resolution is the FWHM of the experimentally measured line spread function.
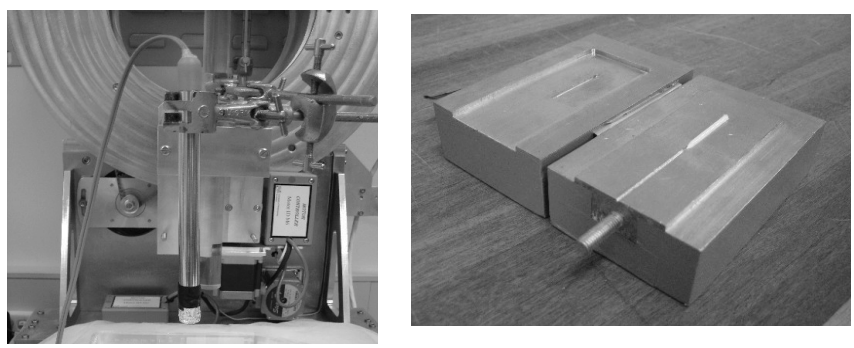

Fig. 2 Photo of (a) the dual probe mounted on the scanning gantry and (b) the two piece lead collimator used in the experiments.

\section{Results and Discussion}

Two different geometries of dual concentric photodiodes were manufactured and investigated. One dual PD had an inner and outer diameter of 3 and $6 \mathrm{~mm}$, respectively. The other dual PD had a $6 \mathrm{~mm}$ inner diameter and a square 10x10 $\mathrm{mm}^{2}$ outer PD design. The results presented in this article focus on the $3 \mathrm{~mm} 6 \mathrm{~mm}$ geometry photodetector.

One important issue investigated here is signal crosstalk. In this case charge created within the diameter of the inner detector may be registered in the outer detector. Such crosstalk can lead to much confusion when carrying out pulse height analysis techniques. To investigate the significance of cross talk in our detector we exposed the photodetector to a $0.5 \mathrm{~mm}$ collimated I-125 source. Fig. 3 shows the response of the inner Si PD to photons from I-125 source (21 and 27 $\mathrm{keV}$ are the main peaks observed) measured with an inhouse designed miniature charge sensitive preamplifier respectively, under $25{ }^{0} \mathrm{C}$. The two main lines are clearly observed and resolved when the collimated source was exposed to the inner photodetector. However when the collimated source is moved onto the outer detector an insignificant number of counts are measured on the inner detector.

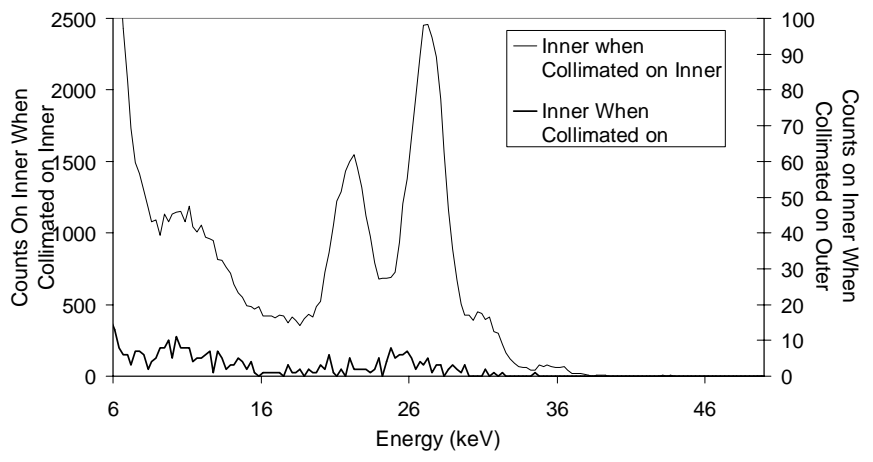

Fig. 3 The response of inner $6 \mathrm{~mm}$ PD on I-125.

The results clearly indicate that the level of cross-talk between the inner and the outer detector is insignificant. What few counts are measured are most likely due to the imperfect collimation of the source. It should also be noted that the cross-talk results displayed here were independently verified using a much more accurate technique (Ion Beam Induced Charge Collection), however this is not discussed here.

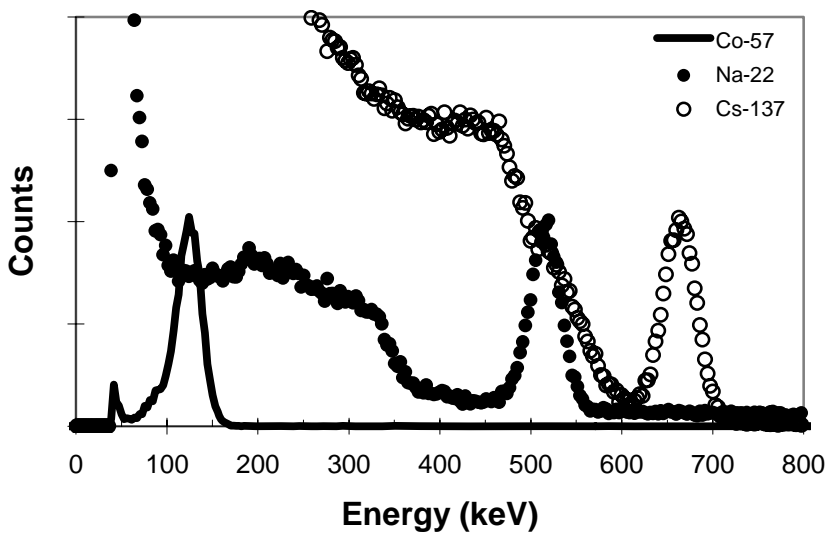

Fig. 4 The response of the inner scintillator detector of the module with a $3 \mathrm{~mm}$ diameter core optically coupled to $\mathrm{CsI}(\mathrm{Tl})$ excited by $122 \mathrm{keV}$ photons from a Co-57 source, $511 \mathrm{keV}$ photons from a $\mathrm{Na}-22$ source and $662 \mathrm{keV}$ photons from a Cs-137 source. 


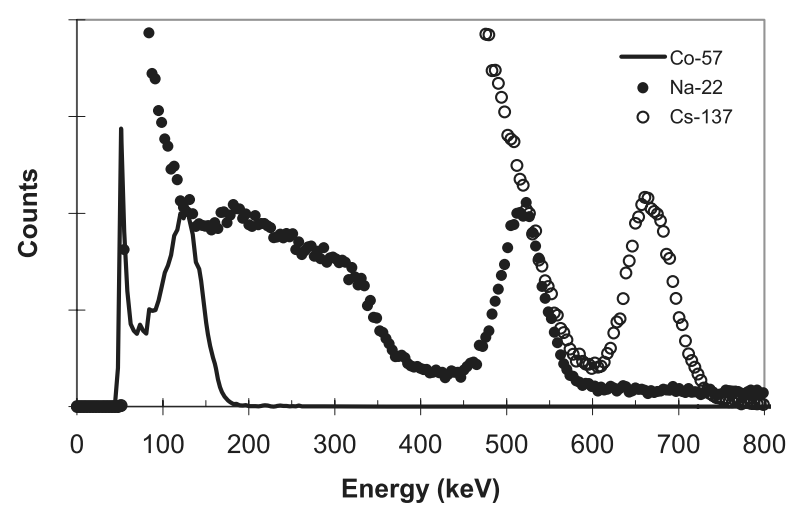

Fig. 5 The response of the outer scintillator detector of the module with a $6 \mathrm{~mm}$ diameter core optically coupled to $\mathrm{CsI}(\mathrm{Tl})$ excited by $122 \mathrm{keV}$ photons from a Co-57 source, $511 \mathrm{keV}$ photons from a $\mathrm{Na}-22$ source and $662 \mathrm{keV}$ photons from a Cs-137 source.

Fig. 4 and 5 show the response of the inner and outer PD design coupled to a dual $\mathrm{CsI}(\mathrm{Tl})$ scintillator excited by collimated gamma sources $\mathrm{Co}-57, \mathrm{Cs}-137$ and $\mathrm{Na}-22$ measured under the same experimental conditions respectively. The ADC was set to 1024 channels for these two spectra. These results may be improved by better geometry of the scintillator, improved optical coupling of the scintillator and optimization of the antireflective coating for $540 \mathrm{~nm}$ light. Taking into account that gap between annular photodiodes is about $0.3 \mathrm{~mm}$ any small misalignment also can lead to a reduction of the number of photons reaching the active area of the photodiode. Special technology of alignment of the scintillator with the PD is currently under development.

Table 1 shows the measured energy resolution of the two concentric scintillator-PD detectors that make up the probe. The data indicates that the probe can be utilized with both low energy and high energy gamma emitting isotopes. The inner detector displays the best energy resolution, which is important in the case where the probe is used in high spatial resolution mode. In this case the counts measured in the outer probe are discarded. Excellent energy resolution is not a high priority, but is important when the probe is used in anti-Compton suppression mode.

Table. 1 Measured energy resolution of the inner and outer detectors that make up the probe.

\begin{tabular}{|cccc|}
\hline & $\mathrm{Cs}-137$ & $\mathrm{Na}-22$ & $\mathrm{Co}-57$ \\
\hline $\begin{array}{c}\text { Energy Resolution [\%] } \\
\text { Inner Detector }\end{array}$ & $5 \pm 3$ & $6 \pm 3$ & $25 \pm 5$ \\
\hline $\begin{array}{c}\text { Energy Resolution [\%] } \\
\text { Outer Detector }\end{array}$ & $10 \pm 3$ & $10 \pm 3$ & $33 \pm 5$ \\
\hline
\end{tabular}

Fig. 6 shows the spatial resolution of the CMRP probe in comparison with a current commercial probe. In this experiment a collimated Tc-99m source was used and the CMRP probe was used in high spatial resolution mode. In this case only the counts from the inner scintillation detector were used and the spatial resolution of the CMRP probe is clearly superior. The spatial resolution is determined by the geometrical configuration of the probe.

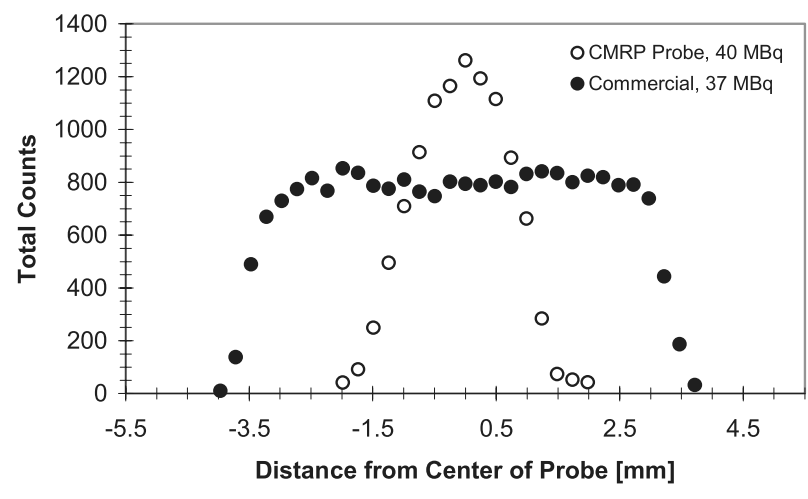

Fig. 6 Measured linear spatial resolution of the CMRP probe in comparison with a current commercial probe in a Tc-99m radiation field.

We also investigated the angular resolution of the CMRP probe in comparison to the same commercial probe. In this case the tip of the probe was placed at the centre of rotation of the collimated source. Fig. $7 \mathbf{a}$ and $7 \mathbf{b}$ show the results of this experiment for two separate source activity levels. The CMRP probe displays better angular resolution for both source activity levels used.
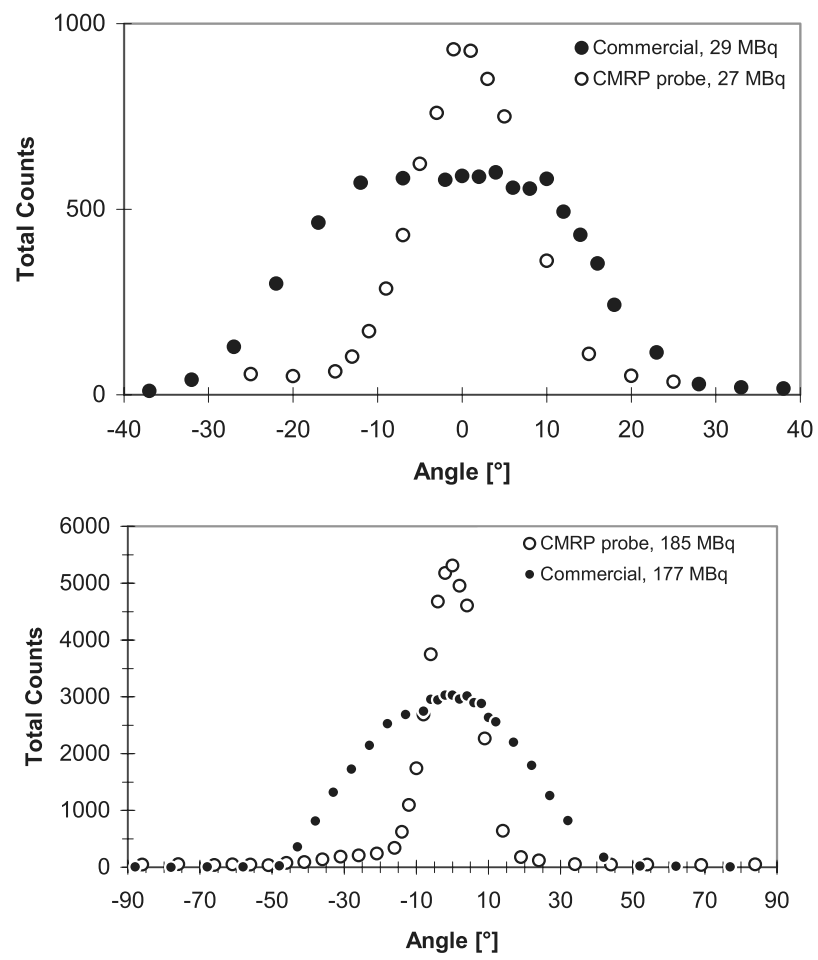

Fig. 7 Measured spatial resolution of the CMRP probe in comparison with a standard, commercially available probe using a relatively (a) weak and (b) strong Tc-99m source.

The final experiment carried out in this study investigated the Compton suppression capabilities of the CMRP detector probe. Fig. 8 shows the effect of running the probe in antiCompton mode with a Cs-137 source. Without Compton 
suppression the level of Compton contribution to the measured pulse height spectrum is clearly much more significant. Such Compton suppression may have very valuable contribution if dual energy radiotracers are utilized as part of the radioguided surgery procedure.

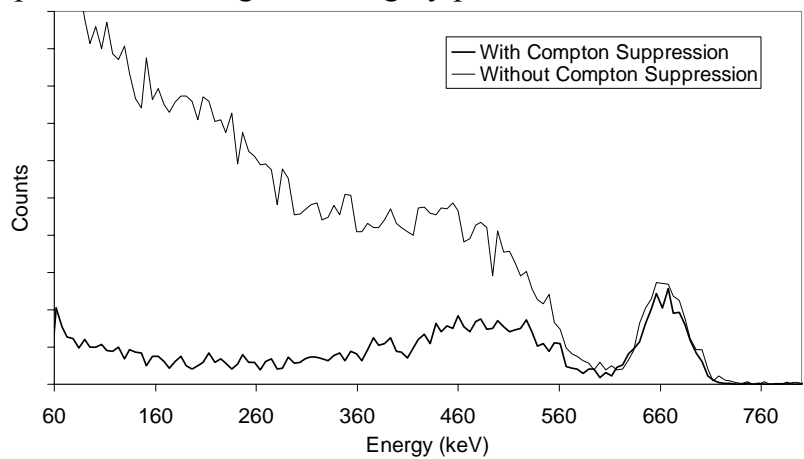

Fig. 8 Measured pulse height spectrum of a Cs-137 source with the CMRP probe operated with and without Compton suppression mode.

\section{Conclusions}

We have characterized the readout performance of a new dual photodetector, dual scintillator probe for radioguided surgery. The obtained energy resolution is enough to realize beta probe in count mode for use with PET isotopes using weighted counts from the outer detector for substraction of gamma $511 \mathrm{keV}$ background from the core detector. In contrast to Si back-to-back detector module ${ }^{3)}$ the sensitivity to $511 \mathrm{keV}$ gamma photons is much higher and can be used additionally to increase the sensitivity of the probe using the core detector for beta and gamma radiations simultaneously. In this case counts due to scattered $511 \mathrm{keV}$ gamma photons will be eliminated using a coincidence technique.

Study with collimated gamma beam demonstrated that no cross talk effect related to charge division between two PDs or light penetration between two concentric scintillators. Readout front-end electronics including shaper amplifier for both channels placed on $10 \times 25 \times 0.2 \mathrm{~mm}$ PC board and antiCompton application of the probe will be investigated and presented along with detailed characterization of proposed module.

\section{Refferences}

1) T.S.Hionkernel et al "Dual detector for surgical tumor staging", J.Nucl. Med. , 29, 1101, (1988).

2) F.Daghighian, E.J.Hofman et al "Intraoperative beta probe: a device for detecting tissue labelled with positron or electron emitting isotopes during surgery", Med Phys, 21, 153, (1994).

3) R.R.Raylman et al "A Dual surface barrier detector unit for beta sensitive endoscopic probes", IEEE Trans on Nucl Sci., 51, N1, 117,(2004). 